\title{
Differentiation of intrafusal fibers from human induced pluripotent stem cells
}

Alisha Colón, Agnes Badu-Mensah, Xiufang Guo, Arindom Goswami, James J. Hickman*

\section{Supplementary Materials}

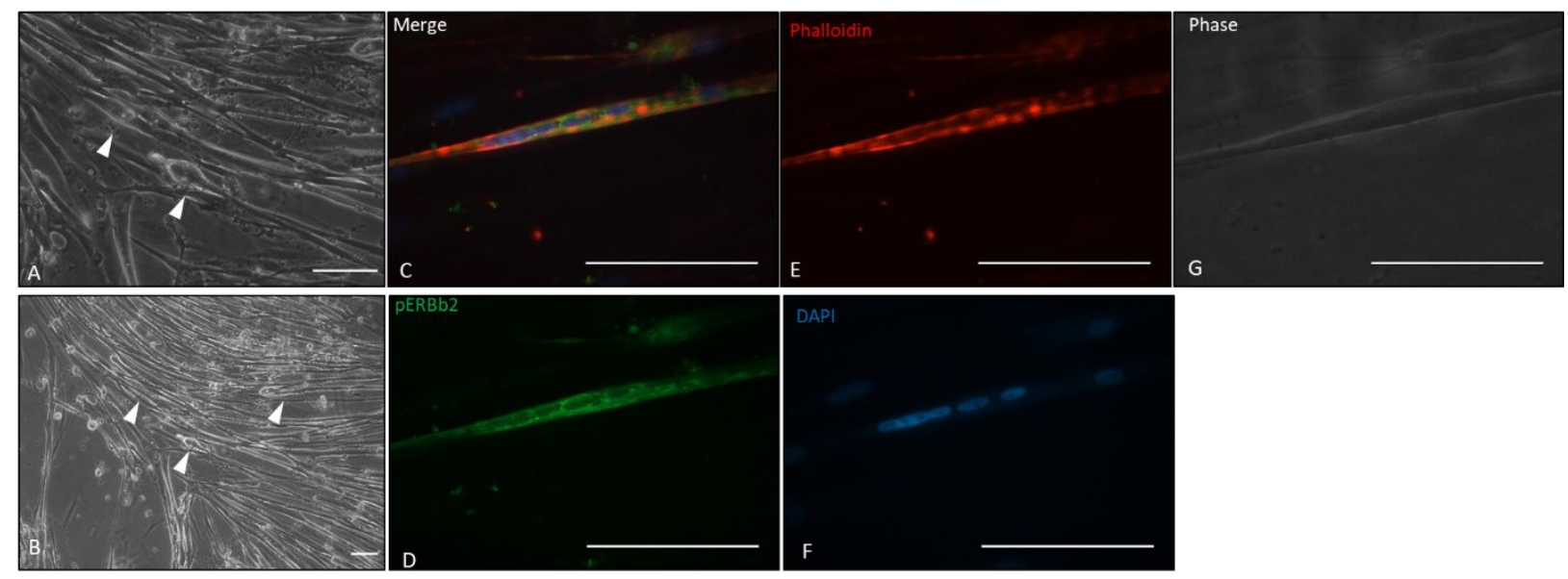

Supplemental Figure 1: Analysis of intrafusal fibers derived from satellite cells. Morphological and immunocytochemical analysis of satellite cell derived intrafusal fibers. A-B) Phase contrast imaging of satellite cell derived intrafusal fibers. White arrowheads show cells with intrafusal morphology. C-G) Immunocytochemistry of satellite cell derived intrafusal fibers. All scale bars are 100 microns. 
Protocol for Myoblast Differentiation from iPSC: Human induced pluripotent stem cell (iPSCs) control line ND41865 was obtained from Coriell Institute for Medical Research. Cells from passages 6 to 12 were used in myoblast differentiation experiments. iPSCs were plated on Matrigel-coated (Cat\# 354230 Corning Life Sciences) surfaces in TESR-E8 culture medium (Cat\# 05990, StemCell Technologies) to a 15$20 \%$ confluence before commencing myoblast differentiation. Myoblast differentiation was performed strictly following a protocol published by Chal $2016^{1}$. .Briefly, iPSC cultures were initially treated with serum-free medium containing insulin-transferrin-selenium (ITS), CHIR-99021 and LDN-193189. On the third day, fibroblast growth factor (FGF) was added to the medium and maintained for three more days. On the sixth day, cultures were switched to medium containing knockout serum replacement (KSR), hepatocyte growth factor (HGF), insulin-like growth factor (IGF), FGF and LDN-193189 for two days. On the eighth day, cultures were fed with medium supplemented with KSR and IGF and maintained in this medium for four days. On the 12th day, HGF was added to the previous medium formulation. Cultures were maintained in the latter medium until 35-40 days of differentiation. Specific concentrations of each factor has been extensively discussed by Chal et al. ${ }^{1}$ Myoblast cultures were then harvested using TrypLE Express (Invitrogen, cat. no. 12605010) and plated at lower densities for morphological and myogenic characterization. After characterization, myoblasts were re-plated for expansion in 1000mg/L DMEM supplemented with MyoCult serum-free medium (Cat \#05982; 05982 StemCell, Technologies). Myoblast were harvested and cryopreserved after $50-60 \%$ confluence in T-75 flasks. All cultures were maintained in humidified air supplemented with $5 \% \mathrm{CO} 2$, at $37^{\circ} \mathrm{C}$.

1. Chal, J., Z. Al Tanoury, M. Hestin, B. Gobert, S. Aivio, A. Hick, T. Cherrier, A.P. Nesmith, K.K. Parker, and O. Pourquie, Generation of human muscle fibers and satellite-like cells from human pluripotent stem cells in vitro. Nat Protoc, 2016. 11(10): p. 1833-50. 